\title{
Functional form comparison between the population and the individual Poisson based TCP models
}

\author{
Colleen Schinkel ${ }^{1,2}$, Nadia Stavreva ${ }^{2}$, Pavel Stavrev ${ }^{2}$, \\ Marco Carlone ${ }^{2,3}$ and B. Gino Fallone ${ }^{1-3}$
}

\author{
${ }^{1}$ Department of Physics, University of Alberta, ${ }^{2}$ Department of Medical Physics, \\ Cross Cancer Institute; ${ }^{3}$ Department of Oncology, University of Alberta, Edmonton, Alberta, Canada
}

In this work, the functional form similarity between the individual and fundamental population TCP models is investigated. Using the fact that both models can be expressed in terms of the geometric parameters $\gamma_{50}$ and $D_{50}$, we show that they have almost identical functional form for values of $\gamma_{50} \geq 1$. The conceptual inadequacy of applying an individual model to clinical data is also discussed. A general individual response TCP expression is given, parameterized by $D_{f}$ and $\gamma_{f}$ - the dose corresponding to a control level of $f$, and the normalized slope at that point. It is shown that the dose-response may be interpreted as an individual response only if $\gamma_{50}$ is sufficiently high. Based on the functional form equivalency between the individual and the population TCP models, we discuss the possibility of applying the individual TCP model for the case of heterogeneous irradiations. Due to the fact that the fundamental population TCP model is derived for homogeneous irradiations only, we propose the use of the EUD, given by the generalized mean dose, when the fundamental population TCP model is used to fit clinical data.

Key words: radiotherapy dosage; Poisson distribution; dose-response relationship, models, statistical, TCP

\section{Introduction}

In the decades following the introduction of the first individual TCP model by Munro and Gilbert, ${ }^{1}$ the distinction between the individual and population response has often been disregarded and individual TCP models have been fit to clinical datasets. The necessity of describ-

Received 01 June 2007

Accepted 20 June 2007

Correspondence to: B. Gino Fallone, Ph.D., Department of Medical Physics, Cross Cancer Institute, 11560 University Ave., Edmonton, Alberta, T6G 1Z2, Canada. Tel: (780) 432-8750, Fax: (780) 432-8615; Email: ginofall@cancerboard.ab.ca, ing the impact of population heterogeneity on dose-response has lead to the development, by a number of authors, of population-based tumour control probability (TCP) models. ${ }^{2-5}$

It has been shown that the presence of population heterogeneity leads to a doseresponse curve that is flattened relative to the individual dose-response curve. If an individual TCP model is fit to a population dataset, the biological meaning of the parameter estimates is lost - the radiobiological parameters take on unrealistically low values. ${ }^{6}$ Nevertheless, although it is conceptually incorrect, the individual TCP model has been fit to clinical datasets and 
parameters obtained from these fits have been assumed to have radiobiologically meaningful values. ${ }^{4,7-10}$ On the other hand, it has also been shown that these fits are characterized by an acceptable goodness of fit.

It has been expected that the population TCP models would allow for the estimation of biologically meaningful population parameters. Unfortunately, it is impossible to obtain a unique set of parameter values when a population TCP model is fit to clinical data. 6,11 This is due to the fact that different sets of population parameter values produce almost identical TCP curves. Carlone et al. ${ }^{11}$ analytically demonstrated that when the dominant source of interpatient heterogeneity is that of tumour radiosensitivity, the population TCP function has only two independent parameters - the dose at $50 \% \mathrm{TCP}, D_{50}$, which determines the position of the TCP curve, and the normalized slope of the curve, $\gamma_{50}$. These parameters have geometric meaning. Since it is also true that the individual TCP model may be expressed in terms of the same two parameters, ${ }^{3,12}$ it is possible that, for a given range of parameter values, both models will exhibit almost identical functional form. In this work, we investigate the similarities between these two models expressed in terms of $D_{50}$ and $\gamma_{50}$ by plotting them for identical values of these geometric parameters.

\section{Background and method}

The general form of the population-based Poisson TCP model has eight parameters. However, it has previously been shown ${ }^{6,11}$ that the parameters of such a model are interrelated; many different combinations of parameters lead to one and the same TCP curve. Thus, it may seem difficult to directly compare the functional forms of the individual and population-based TCP models. On the other hand, Carlone et al. ${ }^{11}$ have specified (based on a certain approximation, of course, but a clinically valid one) what these interrelations actually are, and have shown that there are only two independent population model parameters - $D_{50}$ and $\gamma_{50}$. Fortunately, the individual Pooison-based TCP module can also be parameterized by these parameters. This fact makes the comparison of both models an easier task.

\section{The Poisson-based individual TCP model}

This common form of the individual TCP model is based on Poisson statistics combined with a simplified description of clonogen repopulation. ${ }^{4,10,11,13-26}$ In the case where a tumour undergoes homogeneous irradiation to a total dose $D$, split into $n$ fractions with equal dose per fraction, $d$, the individual Poisson TCP model may be written as: ${ }^{11}$

$$
T C P_{\text {ind }}=e^{-N_{S}}=\exp \left[-N_{0} e^{-(\alpha+\beta d) D+\lambda T}\right]=\exp \left[-N_{0} e^{-\left(\alpha+\beta d-\frac{\lambda^{\prime}}{d}\right) D}\right]=\exp \left[-N_{0} \exp \left(-\alpha^{\prime} D\right)\right],
$$

where $N_{0}$ is the initial number of clonogens, $N_{S}$ is the mean number of clonogens surviving the treatment, $\alpha$ and $\beta$ are the linear quadratic (LQ) radiosensitivity parameters, $\lambda$ is the tumour repopulation rate, $T$ is the total treatment time and $\lambda^{\prime}=\lambda(T / n)$. Note that as long as an equal dose is given during each fraction of the treatment (which is common clinical practice), the parameters $\alpha, \beta$ and $\lambda^{\prime}$ can be combined into one single parameter: 
[2] $\quad \alpha^{\prime}=\alpha+\beta d-\frac{\lambda^{\prime}}{d}$.

The validity of the Poisson TCP model was questioned by Tucker and Travis, ${ }^{21}$ and others ${ }^{27-}$ ${ }^{31}$ who explored the non-Poisson nature of the TCP in the case where tumour repopulation occurs. Under certain conditions, however, it has been shown ${ }^{27,32}$ that the distribution of the number of clonogen cells remaining at the end of a treatment is well-approximated by the Poisson distribution. In view of these results, and also because of the relative complexity of the non-Poissonian TCP models, the individual TCP function presented in Eq. [1] is often used.

A form of the individual TCP model ${ }^{3,12}$ that is equivalent to Eq. [1], but written in terms of the geometric parameters, $\gamma_{50}$ and $D_{50}$, is given by:

$$
\text { [3a] } T C P_{\text {ind }}=0.5^{\exp \left[\frac{2 \gamma_{50}}{\ln 2}\left(1-\frac{D}{D_{50}}\right)\right]} .
$$

The notion of normalized slope, $\gamma$, was first introduced by Brahme ${ }^{33}$ for the purpose of dosimetric precision quantification. Later, Kallman et al. ${ }^{34}$ used the maximum value of $\gamma$ at the inflection point of the TCP curve and derived an expression similar to Eq. [3a], but as pointed out by Bentzen and Tucker, ${ }^{35}$ a slight inconsistency is present in their formula. In general, the Poisson TCP expression given by Eq. [1], may be transformed and parameterized in terms of the normalized slope $\gamma_{f}$ at any dose point $D_{f}$ :

[3b] TCP $P_{\text {ind }}=f^{\exp \left[\frac{-\gamma_{f}}{f \ln f}\left(1-\frac{D}{D_{f}}\right)\right]}$

From Eqs. [1] and [3b], the following relationships between the two different sets of parameters $\left(\gamma_{f}, D_{f}\right)$ and $\left(N_{0}, \alpha^{\prime}\right)$ may be derived:

[4a] $D_{f}=\frac{1}{\alpha^{\prime}} \ln \left(\frac{-N_{0}}{\ln f}\right)$

[4b] $\gamma_{f}=-f \ln f \ln \left(\frac{-N_{0}}{\ln f}\right)$.

and for $\left(\gamma_{50}, D_{50}\right)$ in particular:

[5a] $\quad D_{50}=\frac{1}{\alpha^{\prime}} \ln \left(\frac{N_{0}}{\ln 2}\right)$

[5b] $\quad \gamma_{50}=\frac{\ln 2}{2} \ln \left(\frac{N_{0}}{\ln 2}\right)$.

\section{The population-based TCP model}

Carlone et al. ${ }^{11}$ showed that the population TCP model for the case of dominant heterogeneity in radiosensitivity may be written as:

[6] $T C P_{p o p}=\frac{1}{2} \operatorname{erfc}\left[\sqrt{\pi} \gamma_{50}\left(\frac{D_{50}}{D}-1\right)\right]$. 
The parameters in Eq. [6] $-D_{50}$ and $\gamma_{50}$ - have the same geometric meaning as the corresponding parameters in Eq. [3a]. The geometric parameters may be expressed in terms of the population-based radiobiological parameters, $\overline{\alpha^{\prime}}, \sigma^{\prime}$ and $\overline{\ln N_{0}}: 11$

[7a] $\quad D_{50}=\frac{\Gamma+\ln N_{0}}{\overline{\alpha^{\prime}}}$

[7b] $\gamma_{50}=\frac{\overline{\alpha^{\prime}}}{\sqrt{2 \pi} \sigma^{\prime}}$

Here $\overline{\alpha^{\prime}}=\bar{\alpha}+\bar{\beta} d+\frac{\overline{\lambda^{\prime}}}{d}$ and $\left(\sigma^{\prime}\right)^{2}=\sigma_{\alpha}{ }^{2}+d^{2} \sigma_{\beta}{ }^{2}+\frac{\sigma_{\lambda^{\prime}}{ }^{2}}{d^{2}}$ where $\bar{\alpha}, \bar{\beta}, \overline{\lambda^{\prime}}$ and $\overline{\ln N_{0}}$ are the population averages of the corresponding individual parameters and $\sigma_{\alpha}, \sigma_{\beta}, \sigma_{\lambda^{\prime}}$ and $\sigma_{\ln N_{0}}$ are their standard deviations. The symbol $\Gamma$ represents Euler's gamma constant, which has an approximate value of 0.577 .

The general form of the Carlone et al. ${ }^{11}$ population TCP model takes both heterogeneity in radiosensitivity and heterogeneity in clonogen number into account. However, this form of the population TCP model has three parameters, and was shown ${ }^{11}$ to be almost identical to the one that only takes heterogeneity in radiosensitivity into account. Hence, the latter will be used for this analysis.

\section{Functional form comparison between individual and population-based TCP models}

Since both the individual and the population TCP models may be written in terms of the same two parameters, $\gamma_{50}$ and $D_{50}$, it seems natural to assume that the two models may display similarity in functional form. In order to explore the functional similarity of these models, Eqs. [3a] and [6] are evaluated for a given range of $\gamma_{50}$ and $D_{50}$ values. Subsequently, the individual and population TCP curves obtained for equal sets of $\gamma_{50}$ and $D_{50}$ values are plotted for visual comparison.

The functional closeness of the individual and the population TCP curves may be more rigorously estimated by calculating the normalized difference between the areas under the two TCP curves,

$$
\frac{\Delta A}{A_{T C P_{p o p}}}\left(\gamma_{50}\right)=\frac{\left(A_{T C P_{p o p}}-A_{T C P_{i n d}}\right)}{A_{T C P_{p o p}}},
$$

as a function of $\gamma_{50}$.

\section{Results}

The individual and the population TCP curves were calculated according to Eqs. [3a] and [6] for values of the parameters $\gamma_{50}$ and $D_{50}$ reported by Okunieff et al. ${ }^{36}$ Based on their estimates of $\gamma_{50}$, we chose a range of $\gamma_{50} \in[0.5,6]$. These authors also reported a mean $D_{50}$ for all tumours investigated in their work of $50 \mathrm{~Gy}$, with values ranging from 10 to $90 \mathrm{~Gy}$. We therefore chose a value of $D_{50}=50 \mathrm{~Gy}$ for our investigation.

Figure 1 shows eight pairs of individual and population TCP curves calculated for the following parameter values: $D_{50}=50 \mathrm{~Gy}$ and $\gamma_{50}=[0.5,1,1.5,2,2.5,3,4,6]$. This figure was reproduced for different values of $D_{50}$, to determine whether this parameter 
had any influence on functional equivalency. It was found that the location of the TCP curves along the dose-axis did not influence the shapes of the curves or their positions relative to each other. Hence, the results shown in Figure 1 are applicable for any $D_{50}$ value.

The quantity $\frac{\Delta A}{A_{T C P_{p o p}}}\left(\gamma_{50}\right)$ (Eq. [8]) is plotted in Fig. 2. The largest area difference between the two TCP curves is $-17.7 \%$ obtained at $\gamma_{50}=0.5$.

\section{Discussion}

Based on Figures 1(d) - 1(h) and Figure 2, one may conclude that the functional forms of the individual and the population models are almost identical for $\gamma_{50} \in[2,6]$. Indeed, for this range of $\gamma_{50}$ the index $\left|\triangle A / A_{T C P_{p o p}}\right|$ is less than $0.5 \%$. Although $\left|\triangle A / A_{T C P_{p o p}}\right|$ is higher $\left(\triangle A / A_{T C P_{p o p}} \in[-0.5,-6.7] \%\right)$ for the interval $\gamma_{50} \in[1,2)$, the plots in Figures $1(b)$ and $1(\mathrm{c})$ indicate that the individual and population TCP curves are still sufficiently close to each other, especially for the clinically-relevant high dose range. The individual and population models differ considerably at $\gamma_{50}=0.5\left(\left|\Delta A / A_{T C P_{p o p}}\right|=17.7 \%\right)$. As can be seen from Figure 1, for $\gamma_{50}$ less than 2.5 the individual curves overread TCP everywhere except at 50\% control when compared with the population-based TCP curves. For normalized slopes above $\gamma_{50}=$ 2.5, the individual curves tend to slightly underread the population TCP. The overreading and underreading tendencies are clearly demonstrated by Figure 2.
The considerable closeness in functional form of both models explains the observation that the individual TCP model produces a reasonable fit to clinical datasets. ${ }^{4,10}$ In spite of this, the observed equivalence in functional form of the two TCP models should not be regarded as an endorsement to use the individual TCP model to fit clinical data.

However, a very steep dose response is unusual for clinical data sets. Shallower responses are much more typical for populations of patients. Therefore, it would conceptually be more correct to use the population TCP model, which accounts for interpatient heterogeneity to fit such data. If, however, the individual TCP model is used, one should bear in mind that the obtained parameter values have lost their biological meaning and should be interpreted simply as phenomenological coefficients.

As can be seen from Figures 1(a) and $1(b)$, both models start to differ in functional form for the clinically observable range of $\gamma_{50}<1$. In addition, for these values of $\gamma_{50}$, the individual model leads to TCP $>0$ for $D$ $=0$. Therefore, fits to very shallow curves using the individual model may distort the best-fit estimates of $\gamma_{50}$ and $D_{50}$.

The authors advocate the use of the population model in regards to clinical data. However, the demonstrated equivalence in functional form of the individual and population models can be utilized for the case of heterogeneous tumour irradiation. In this case, the individual TCP model with existing $\left\{\gamma_{50}, D_{50}\right\}$ estimates (e.g. Okunieff et al. ${ }^{36}$ ) can be used for the evaluation of $\mathrm{TCP}^{37}$ according to the following expression: ${ }^{38}$

$$
\text { [9] } \quad T C P=0.5^{\sum^{i} v_{i} \exp \left[\frac{2 \gamma_{50}}{\ln 2}\left(1-\frac{D_{i}}{D_{50}}\right)\right]}
$$

Equation [9] is a simple, straightforward generalization of Eq. [3] for the case of heterogeneous irradiation. The generalization of Eq. [6] for the case of heterogeneous irradiation, without introducing extra model pa- 

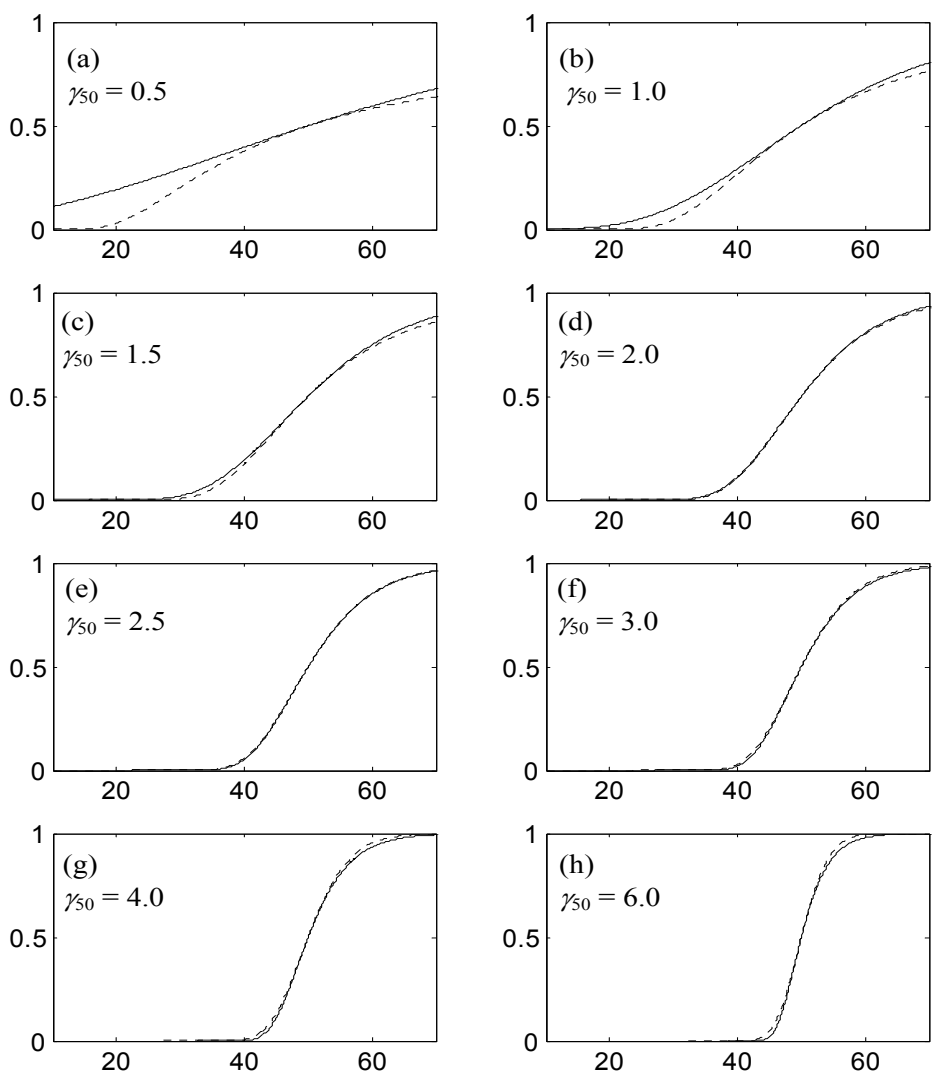

Dose (Gy)

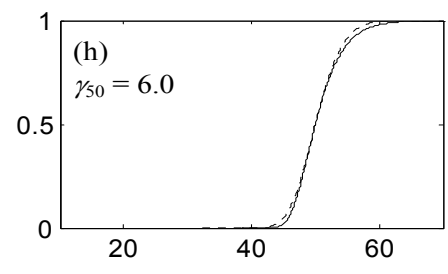

Dose (Gy) mean dose (GMD), as is usually done. ${ }^{39,40}$ Unfortunately, this approach introduces a third model parameter, and knowledge of its value for each tumour type would then be needed in order to use this model to calculate TCP for a heterogeneously irradiated tumour. Therefore, until more comprehensive parameter estimates are produced through fits of the population TCP model to clinical data for the case of heterogeneousirradiation, we propose that Eq. [9] be used for evaluation of treatment plans in terms of TCP, based on the functional form equivalency of both models.

Figure 1. Individual (solid) and population-averaged (dotted) TCP curves for $\mathrm{D}_{50}=50$ Gy and the $\gamma_{50}$ values shown in each sub-plot.

rameters, presents a complicated mathematical problem, and has not yet been solved.

Strictly speaking, the ability to use Eq. [9] as a population TCP descriptor has not yet been proven theoretically. Nevertheless, our experience with the TCP/NTCP estimation module ${ }^{37}$ shows that it produces reasonable TCP estimates.

Another approach to the problem of taking dose heterogeneity into account for the population TCP model is to replace the homogeneous dose, $D$, with the equivalent uniform dose, EUD. It may then be assumed that the EUD is equal to the generalized

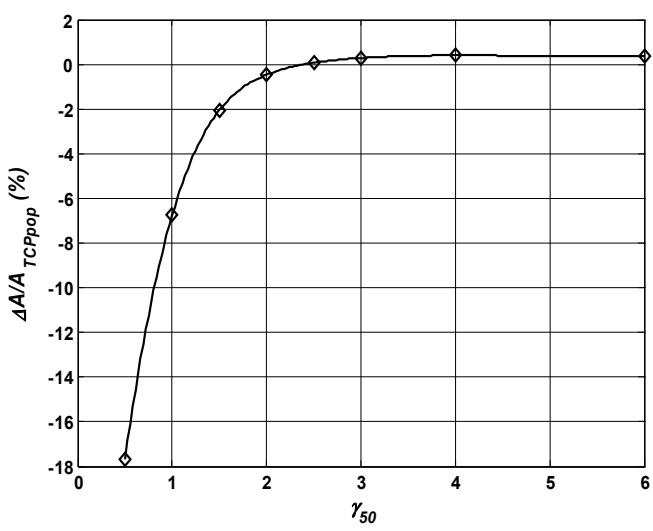

Figure 2. The ratio of the area difference, $\triangle A=A_{\text {TCPроp }}-A_{\text {TCPind }}$, between the two TCP curves, to the total area under the population TCP curve $\left(A_{\text {TСРрор }}\right)$, plotted for the values of $\gamma_{50}$ used to generate the curves shown in Figure 1. 


\section{Conclusions}

It is thus concluded that:

- The population and the individual TCP responses are almost identical in functional form for $\gamma_{50}$ belonging to the interval [1, 6]. If each of these models were fit to the same clinical dataset, they would produce statistically indistinguishable values of the parameters $D_{50}$ and $\gamma_{50}$.

- It is conceptually incorrect to use the individual TCP model to fit clinical data.

- Until reliable estimates of the population TCP parameters for the case of heterogeneous tumour irradiation are obtained, the individual TCP model (Eq. [9]) with existing $D_{50}$ and $\gamma_{50}$ estimates could be used for TCP evaluations in this situation.

- The case of a shallow dose-response relationship, which is usually observed clinically, can be explained by the presence of significant inter-patient heterogeneity. The population TCP model should be used to fit such data, as it accounts for this heterogeneity. If, however, the individual TCP model is used, the estimated parameter values should be interpreted simply as phenomenological coefficients.

- A steep dose-response relationship indicates the presence of a relatively small interpatient heterogeneity. Though it is highly improbable to observe such dose-responses clinically, the individual TCP model may be applied to such data for the purpose of estimating biological parameters, as the individual parameters would retain some biological meaning in this case.

\section{Acknowledgements}

This research was supported by studentships from the Alberta Foundation for Medical Research, the Alberta Cancer Board and the Translational Research Training in
Cancer program (the Canadian Institutes for Health Research), as well as the Alberta Cancer Board Research Initiative Program Grant RI-218.

\section{References}

1 Munro TR, Gilbert CW. The relation between tumour lethal doses and the radiosensitivity of tumour cells. Br J Radio 1961; 34: 246-51.

2 Fenwick JD. Predicting the radiation control probability of heterogeneous tumour ensembles: data analysis and parameter estimation using a closedform expression. Phys Med Biol 1998; 43: 2159-78.

3 Goitein M, Niemierko A, Okunieff P. The probability of controlling an inhomogeneously irradiated tumour: A strategem for improving tumour control through partial tumour boosting. Presented at the 19th L. H. Gray Conference: Quantitative Imaging in Oncology, Newcastle, UK; 1995.

4 Roberts SA, Hendry JH. A realistic closed-form radiobiological model of clinical tumor-control data incorporating intertumor heterogeneity. Int $J$ Radiat Oncol Biol Phys 1998; 41: 689-99.

5 Webb S, Nahum AE. A model for calculating tumour control probability in radiotherapy including the effects of inhomogeneous distributions of dose and clonogenic cell density. Phys Med Biol 1993; 38: 653-66.

6 Warkentin B, Stavrev P, Stavreva NA, Fallone BG. Limitations of a TCP model incorporating population heterogeneity. Phys Med Biol 2005; 50: 357188 .

7 Brenner DJ. Dose, volume, and tumor-control predictions in radiotherapy. Int J Radiat Oncol Biol Phys 1993; 26: 171-9.

8 D'Souza WD, Thames HD, Kuban DA. Dose-volume conundrum for response of prostate cancer to brachytherapy: summary dosimetric measures and their relationship to tumor control probability. Int J Radiat Oncol Biol Phys 2004; 58: 1540-8.

9 Fowler J, Chappell R, Ritter M. Is alpha/beta for prostate tumors really low? Int J Radiat Oncol Biol Phys 2001; 50: 1021-1031.

10 Roberts SA, Hendry JH. The delay before onset of accelerated tumour cell repopulation during radiotherapy: a direct maximum-likelihood analysis of a collection of worldwide tumour-control data. Radiother Oncol 1993; 29: 69-74. 
11 Carlone M, Warkentin B, Stavrev P, Fallone BG. Fundamental form of the population TCP model in the limit of large heterogeneity. Med Phys 2006; 33: $1634-42$.

12 Stavrev P, Stavreva N, Niemierko A, Goitein M. Generalization of a model of tissue response to radiation based on the idea of functional subunits and binomial statistics. Phys Med Biol 2001; 46: 1501-18.

13 Dale RG. Radiobiological assessment of permanent implants using tumor repopulation factors in the linear-quadratic model. Br J Radiol 1989; 62: 241-4.

14 Dale RG. Time-dependent tumour repopulation factors in linear-quadratic equations--implications for treatment strategies. Radiother Oncol 1989; 15: 371-81.

15 Fowler JF. The linear-quadratic formula and progress in fractionated radiotherapy. Brit $J$ Radiol 1989; 62: 679-94.

16 Maciejewski B, Withers HR, Taylor JM, Hliniak A. Dose fractionation and regeneration in radiotherapy for cancer of the oral cavity and oropharynx: tumor dose-response and repopulation. Int J Radiat Oncol Biol Phys 1989; 16: 831-43.

17 Maciejewski B, Withers HR, Taylor JM, Hliniak A. Dose fractionation and regeneration in radiotherapy for cancer of the oral cavity and oropharynx. Part 2. Normal tissue responses: acute and late effects. Int J Radiat Oncol Biol Phys 1990; 18: 101-11.

18 Taylor JM, Withers HR, Mendenhall WM. Dosetime considerations of head and neck squamous cell carcinomas treated with irradiation. Radiother Oncol 1990; 17: 95-102.

19 Thames HD, Bentzen SM, Turesson I, Overgaard M, Van den Bogaert W. Time-dose factors in radiotherapy: a review of the human data. Radiother Oncol 1990; 19: 219-35.

20 Travis EL, Tucker SL. Isoeffect models and fractionated radiation therapy. Int J Radiat Oncol Biol Phys 1987; 13: 283-7.

21 Tucker SL, Travis EL. Comments on a time-dependent version of the linear-quadratic model. Radiother Oncol 1990; 18: 155-3.

22 Van Dyk J, Mah K, Keane TJ. Radiation-induced lung damage: dose-time-fractionation considerations. Radiother Oncol 1989; 14: 55-69.

23 van de Geijn J. Incorporating the time factor into the linear-quadratic model. Brit J Radiol 1989; 62: 296-8.
24 Wheldon TE, Amin AE. The Linear Quadratic Model. Brit J Radiol 1988; 61: 700-2.

25 Withers HR, Taylor JM, Maciejewski B. The hazard of accelerated tumor clonogen repopulation during radiotherapy. Acta Oncol 1988; 27: 131-46.

26 Yaes RJ. Linear-quadratic model isoeffect relations for proliferating tumor cells for treatment with multiple fractions per day. Int J Radiat Oncol Biol Phys 1989; 17: 901-5.

27 Hanin LG, Zaider M, Yakovlev AY. Distribution of the number of clonogens surviving fractionated radiotherapy: a long-standing problem revisited. Int J Radiat Biol 2001; 77: 205-13.

28 Kendal WS. A closed-form description of tumour control with fractionated radiotherapy and repopulation. Int J Radiat Biol 1998; 73: 207-10.

29 Yakovlev A. Comments on the distribution of clonogens in irradiated tumors. Radiat Res 1993; 134: 117-22.

30 Zaider M, Minerbo GN. Tumour control probability: a formulation applicable to any temporal protocol of dose delivery. Phys Med Biol 2000; 45: 279-93.

31 Zaider M, Zelefsky MJ, Hanin LG, Tsodikov AD, Yakovlev AY, Leibel SA. A survival model for fractionated radiotherapy with an application to prostate cancer. Phys Med Biol 2001; 46: 2745-58.

32 Hanin LG. A stochastic model of tumor response to fractionated radiation: limit theorems and rate of convergence. Math Biosci 2004; 191: 1-17.

33 Brahme A. Dosimetric precision requirements in radiation therapy. Acta Radiol Oncol 1984; 23: 37991.

34 Kallman P, Agren A, Brahme A. Tumour and normal tissue responses to fractionated non-uniform dose delivery. Int J Radiat Biol 1992; 62: 249-62.

35 Bentzen SM, Tucker SL. Quantifying the position and steepness of radiation dose-response curves. Int J Radiat Biol 1997; 71: 531-42.

36 Okunieff P, Morgan D, Niemierko A, Suit HD. Radiation dose-response of human tumors. Int $\mathrm{J}$ Radiat Oncol Biol Phys 1995; 32: 1227-37.

37 Warkentin B, Stavrev P, Stavreva N, Field C, Fallone BG. A TCP-NTCP estimation module using DVHs and known radiobiological models and parameter sets. J Appl Clin Med Phys 2004; 5: 50-63. 
38 Niemierko A. Radiobiological models of tissue response to radiation in treatment planning systems. Tumori 1998; 84: 140-3.

39 Choi B, Deasy JO. The generalized equivalent uniform dose function as a basis for intensity-modulated treatment planning. Phys Med Biol 2002; 47: 3579-89.

$40 \mathrm{Wu}$ Q, Mohan R, Niemierko A, Schmidt-Ullrich R. Optimization of intensity-modulated radiotherapy plans based on the equivalent uniform dose. Int $J$ Radiat Oncol Biol Phys 2002; 52: 224-35. 